\title{
Molecular Self-assembly and Ordering of Alkylpolyglycosides for Improved Adhesion in Paper Coatings
}

\author{
L. T. Germinario, C. M. Buchanan and M. D. Wood \\ Eastman Chemical Company, Global Polymers and Research Analytical Services, \\ P.O. Box 1972, Kingsport, TN 37662-5150
}

Cellulose is the most abundant polymer found in nature and in some forms is used as heat-sealable paper for medical packaging applications where it acts as a filter to particulates and microorganisms, while at the same time permitting free passage of gas and water vapor during sterilization. This paper must also be heat-sealable over plastic trays that contain medical instrumentation. The bond of the paper to the plastic must be sufficiently strong, but at the same time capable of being peeled from the plastic tray without tearing. The additive that was found to be suitable as an adhesion promoter was alkylpolyglycoside (APG), a nonionic surfactant, in combination with cellulose acetate fibers (CA). [1] For heat-sealable paper, a mechanism for improved adhesion for APG and CA containing paper was proposed in which APG anchors itself in the cellulosic matrix and acts as a plasticizer for CA. [1] In order to test this hypothesis, Atomic Force Microscopy (AFM) was employed, as it is a non-destructive analytical tool capable of probing three-dimensional surface structures and properties at the nanoscale.

Paper containing $25 \%$ cellulose acetate was coated with a $3 \%$ solution of APG (DP $=1.5$, average alkyl chain length $=9.9$ ). Three-dimensional views of fiber surfaces from coated and uncoated paper (Fig. 1) show distinct regions that contain surface patches that are not present in the corresponding areas of uncoated paper. The heights of surface patches were measured using AFM-based bearing analysis. The boxed region drawn in the image in Fig. 2, depict surface features with average heights of $3.6 \mathrm{~nm}$. In order to establish the thickness of native APG monolayers on atomically flat substrates, Langmuir-Blodgett (LB) techniques were employed. Figure 3, is a top view of an APG film that was deposited on mica from an LB trough with an average thickness of $3.1 \mathrm{~nm}$. As is evident, the thickness of LB films deposited on mica substrates are comparable in thickness to the patch heights measured on coated fibers $(3.6 \mathrm{~nm})$.

In order to gain further insight into potential molecular packing and ordering geometries for APG films, molecular modeling tools were employed (CAChe). APG molecules were created by linking two glucose moieties and a $\mathrm{C} 10$ alkyl chain. A low energy molecular 3D structure was generated and optimized in MOPAC using PM3 parameters. Molecular packing geometries were also explored. The APG molecular structures generated in CAChe suggest the presence of APG bilayers on coated paper, with the alkyl chains buried in the center of the bilayer and glucose moieties aligned along the surface. The computed layer thickness of $3.6 \mathrm{~nm}$ compares favorably with the patch heights experimentally measured by AFM $(3.6 \mathrm{~nm})$, figure 2. This type of ordering of amphiphilic molecules is reminiscent of the classic lipid bilayer structure found in cell membranes, where London dispersion forces stabilize the lipid-lipid chain interactions. [2]

References

[1] C. M. Buchanan and M. D. Wood, Polymer Preprints (ACS) (1998) 84.

[2] J. N. Israelachvili, Intermolecular and Surface Forces, $2^{\text {nd }}$ ed., Academic Press, (1998) 83. 

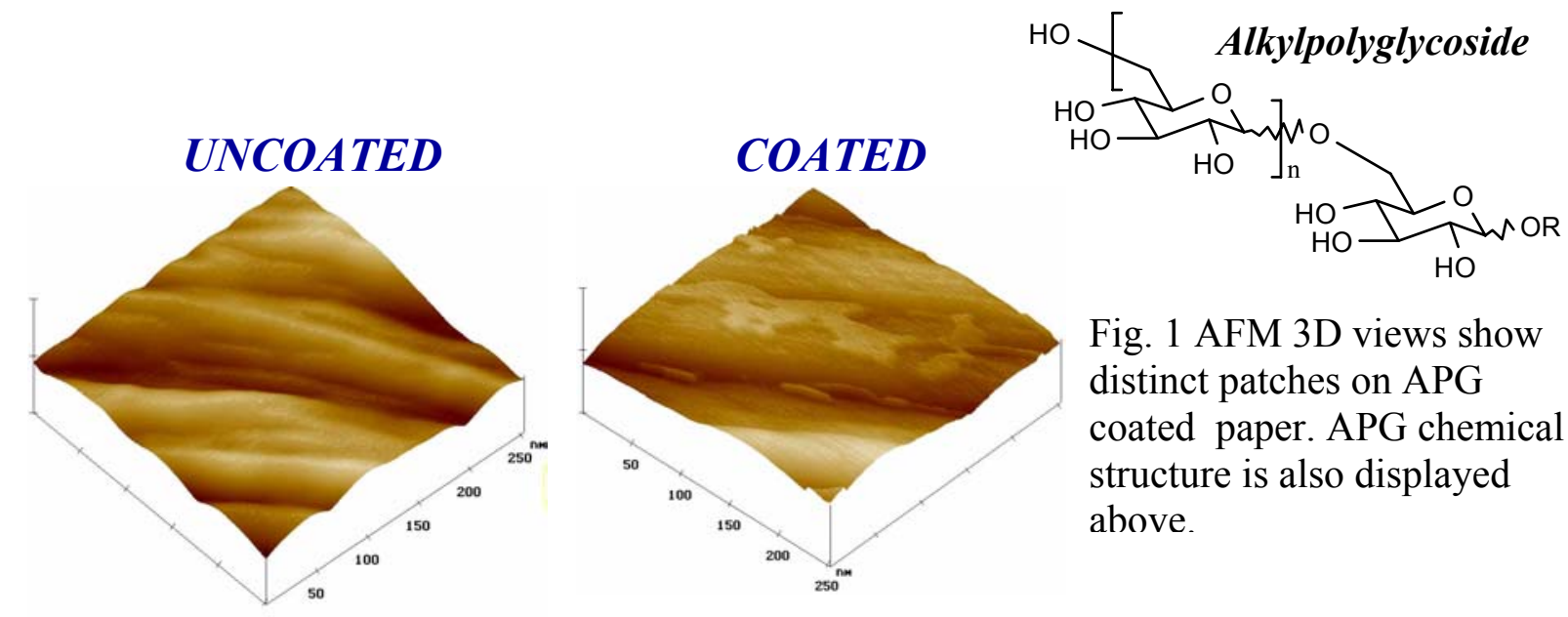

Fig. 1 AFM 3D views show distinct patches on APG coated paper. APG chemical structure is also displayed above.

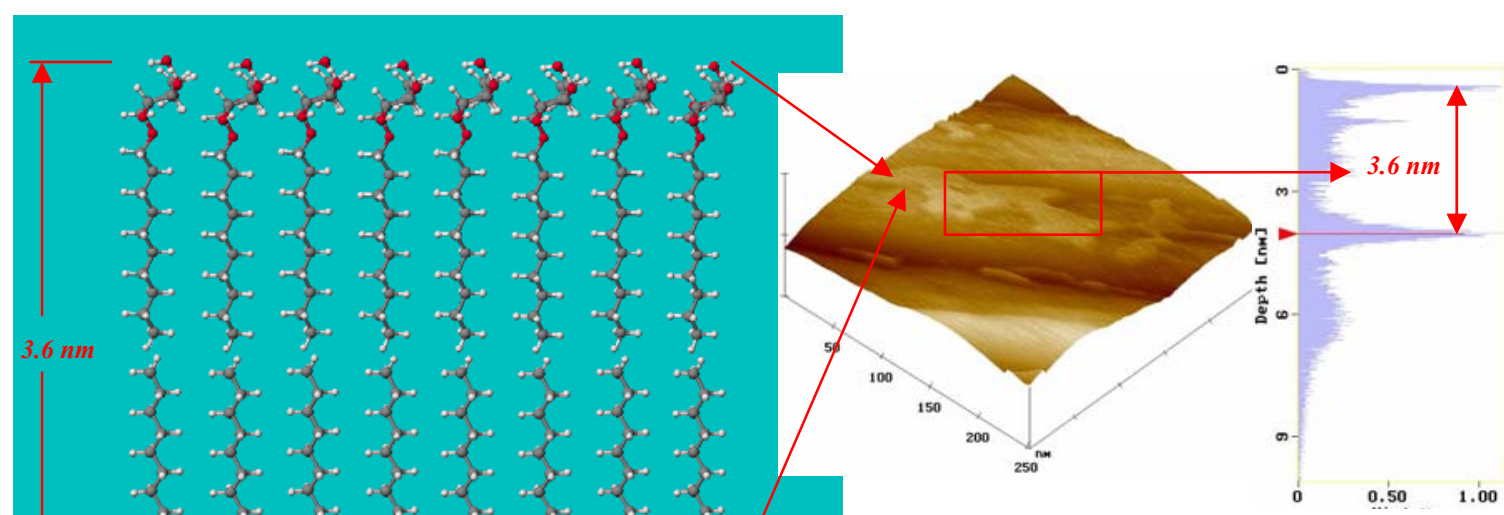

Fig. 2 Bearing analysis show patches with average heights of $3.6 \mathrm{~nm}$. Molecular modeling tools using CAChe provided strong evidence for the presence of a bilayer structure.
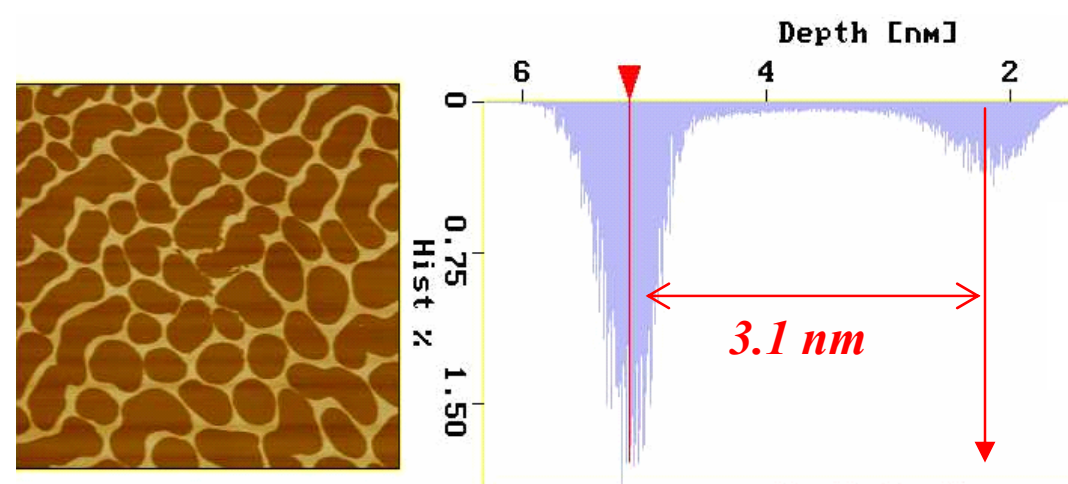

Fig. 3 Bearing analysis of APG deposited on mica from LB trough. 